\title{
The influence of barley $\beta$-glucan on satiety, glycaemic response and energy expenditure
}

\author{
M. E. Clegg and P. S. Thondre \\ Functional Food Centre, Department of Sport and Health Sciences, Oxford Brookes University, Gipsy Lane, Oxford, \\ $O X 3 O B P, U K$
}

The concept of glycaemic response (GR) is now well established as a tool to help in the management of many widespread chronic health conditions $^{(1)}$. Barley $\beta$-glucan forms a highly viscous solution in the stomach and small intestine, which entraps glucose molecules and slows down their intestinal absorption resulting in slower release of blood glucose and increased satiety. The impact of this on energy expenditure remains unknown. The aim of the current study was to compare the GR, diet induced thermogenesis (DIT) and satiety of soups containing either high or low molecular weight (MW) $\beta$-glucan or a control soup containing no $\beta$-glucan.

Eleven healthy volunteers ( 8 female, 3 male; age: 28.6 (SEM 1.9) yr; height: 1.69 (SEM 0.03) m; weight: 70.8 (4.1 SEM) kg) were tested on three occasions following an overnight fast. Volunteers were fed soup equivalent to $50 \mathrm{~g}$ of available carbohydrate portions containing either $3 \mathrm{~g}$ of high MW $\beta$-glucan, $3 \mathrm{~g}$ of low MW $\beta$-glucan or no $\beta$-glucan. Baseline and postprandial GR were measured using finger prick blood samples; satiety was measured using visual analogue scales (VAS) and resting metabolic rate and DIT using an indirect calorimeter. Analysis was completed using repeated measures ANOVA. Statistical significance was set at $P<0.05$.

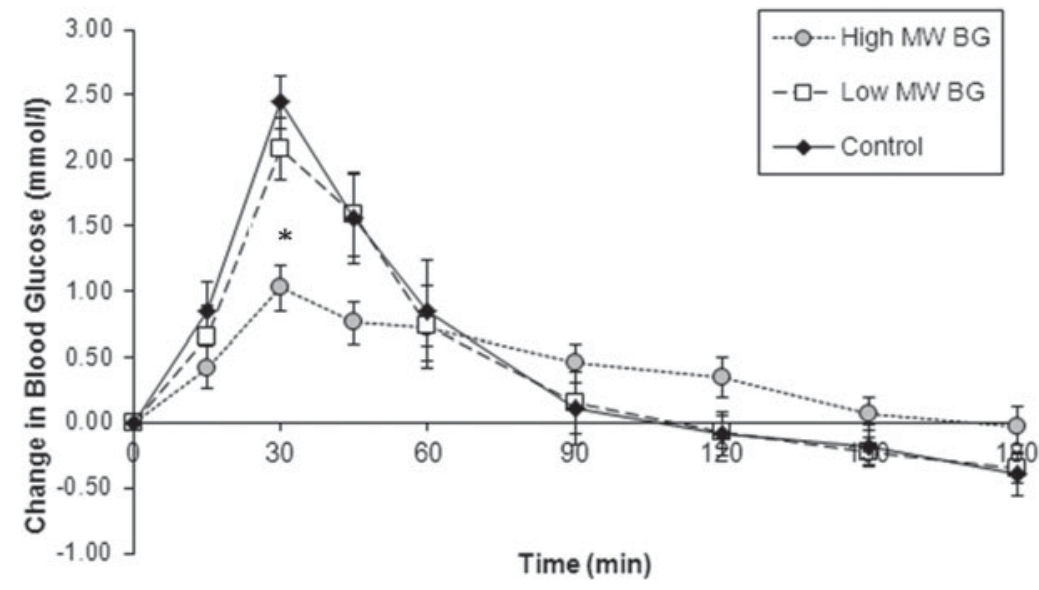

Glycaemic response (mean \pm SEM) following each of the soup meals

The GR for the high MW $\beta$-glucan soup was significantly lower than the control soup and low MW $\beta$-glucan soup at 30 min in the postprandial phase $(P<0.01)$. There were no significant differences in any of the VAS parameters; hunger, fullness, desire to eat and prospective food consumption $(P>0.05)$. For DIT, the difference between the three soups was not significant but was approaching significance $(P=0.064)$. The high MW $\beta$-glucan soup had a significantly lower DIT than the control soup $(P=0.01)$. The difference in DIT between the high and low MW $\beta$-glucan soups also was approaching significance $(P=0.084)$. The highest DIT was for the control soup and the lowest was for the high MW $\beta$-glucan soup.

Previous studies have shown that MW of $\beta$-glucan influences GR of solid foods ${ }^{(2)}$ and total and LDL cholesterol levels ${ }^{(3)}$ with high MW $\beta$-glucan having a beneficial effect compared to low MW $\beta$-glucan. This data indicates that low MW $\beta$-glucan might be more beneficial compared to high MW $\beta$-glucan in increasing DIT.

1. Jenkins DJ, Wolever TM, Taylor RH et al. (1981) Am J Clin Nutr 34, 362-366.

2. Thondre PS \& Henry CJ. (2011) Int J Food Sci Nutr 62, 678-84.

3. Wolever TM, Gibbs AL, Brand-Miller J et al. (2011) Nutr J 25, 130. 